\title{
EARLY LUNG ALLOGRAFT FUNCTION AFTER RETROGRADE AND ANTEGRADE PRESERVATION
}

A. Varela, MD, M. Cordoba, MD, S. Serrano-Fiz, MD, R. Burgos, MD, C. G. Montero, MD, G. Téllez, MD, N. Novoa, MD, E. Castedo, MD, E. Tebar, MD, J. Téllez, MD, J. Roda, MD, and J. Ugarte, MD, Madrid, Spain

Different techniques have been designed to provide adequate lung preservation by antegrade flushing of a preservation solution, generally modified Euro-Collins solution or University of Wisconsin solution, through the pulmonary artery (PA). Despite these pharmacologic advances, early allograft dysfunction remains a problem. An entirely new concept is retrograde instillation of the preservation solution through the left atrial appendage, with the PA used for outflow.

This novel approach has been successfully employed in the laboratory ${ }^{1-3}$ and for 23 consecutive lung transplantation procedures. ${ }^{4}$ The observations and direct implications derived from this limited experience constitute the basis for this report.

Technique. Between January 1996 and January 1997, 23 consecutive lung transplantation procedures (10 uni-

From the Department of Thoracic and Cardiovascular surgery, Hospital Puerta de Hierro, Madrid, Spain.

Received for publication June 11, 1997; accepted for publication June 19, 1997.

Address for reprints: A. Varela, MD, C/San Martin de Porres No. 4, 28035 Madrid, Spain.

J Thorac Cardiovasc Surg 1997;114:1119-20

Copyright (c) 1997 by Mosby-Year Book, Inc. $0022-5223 / 97 \$ 5.00+0 \quad \mathbf{1 2 / 5 4 / 8 4 3 0 1}$ lateral and 13 bilateral) were carried out after lung preservation by means of the "retrograde-antegrade technique" (Fig. 1). This was accomplished with a short-tipped William Harvey cannula (Bard and William Harvey, Santa Ana, Calif.) for instillation of the pneumoplegic solution with modified Euro-Collins solution (Laboratorios Esteve, Barcelona, Spain) through the left atrial appendage, with outflow through the PA.

Cardioplegia had been induced previously with $1 \mathrm{~L}$ crystalloid solution instilled into the aortic root. A $14 \mathrm{~F}$ to $16 \mathrm{~F}$ cannula was positioned in the left atrial appendage for retropneumoplegia. A single bolus of prostaglandin $\mathrm{E}_{1}$ (500 mg) was injected into the PA before flushing (4000 $\mathrm{ml}, 4^{\circ} \mathrm{C}$ ) of the pneumoplegic solution from approximately $30 \mathrm{~cm}$ above table level. The time consumed by this technique was usually about 5 to 8 minutes.

Left ventricular distention was prevented by venting the PA. Both pleural cavities were opened and continuously irrigated with cold saline slush to further enhance graft preservation. The heart was removed and the double-lung block was adequately excised. Before immersion in the transport bag, $500 \mathrm{ml} 4^{\circ} \mathrm{C}$ pneumoplegic solution was carefully instilled into each PA branch. Adequate washing out of all remaining blood to remove fibrin debris was verified in all cases to clear both pulmonary and bronchial capillary networks. Occasionally, the technique has been reversed; that is, antegrade PA flushing is carried out first

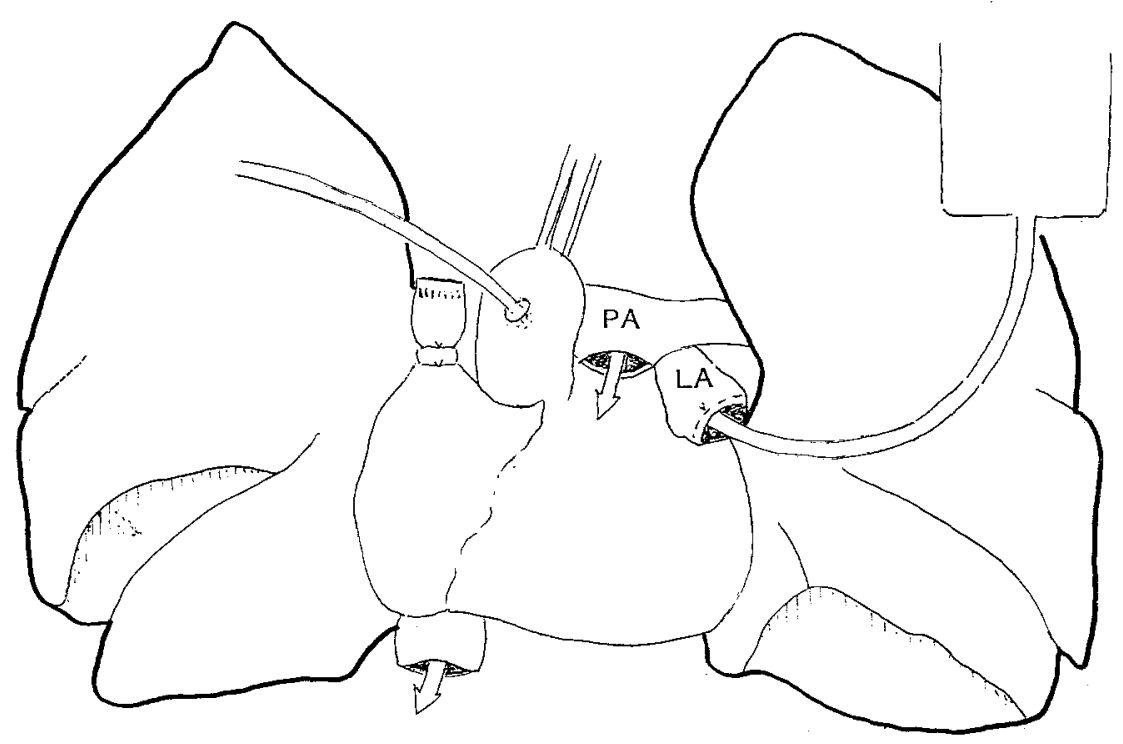

Fig. 1. Biphasic lung graft preservation through the left atrial appendage, venting the PA (first phase). Back-table work includes antegrade (PA) perfusion of the pneumoplegic solution (second phase). LA, Left atrial appendage. Arrows, Inferior vena cava. 


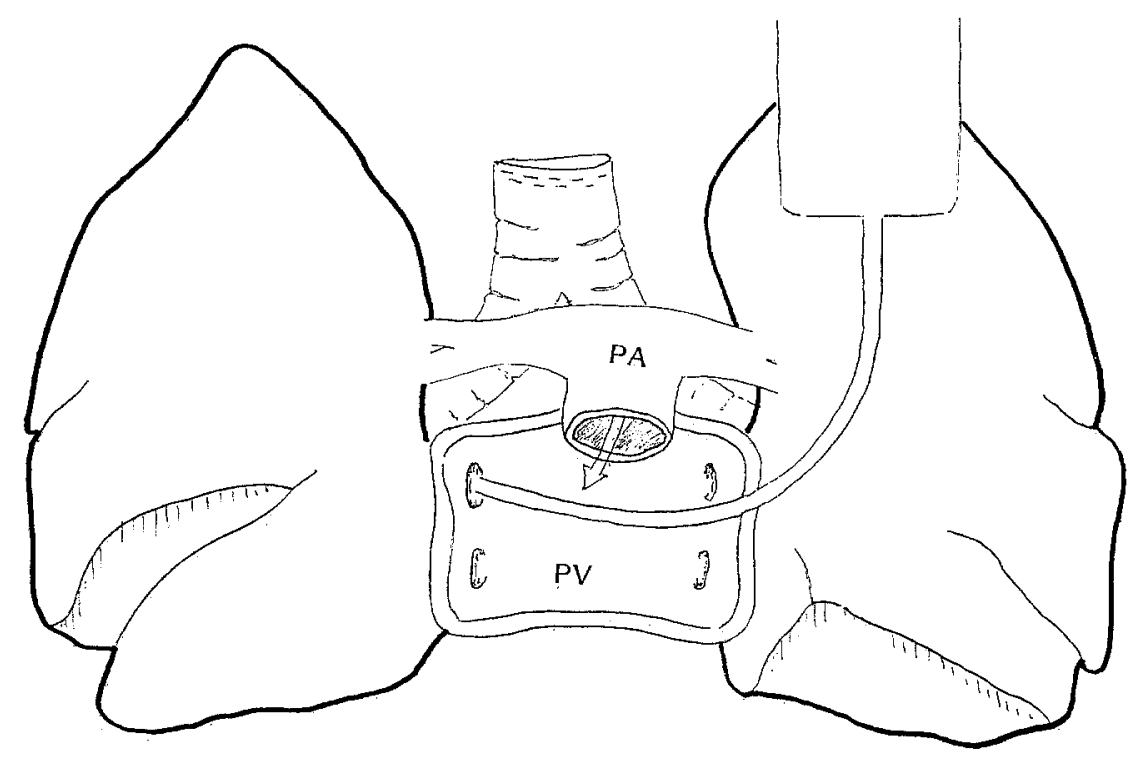

Fig. 2. When antegrade (PA) lung preservation is used, retrograde perfusion through pulmonary veins is completed at the back table. PV, Pulmonary veins. Arrow, Venting PA.

Table I. Early lung allograft function

\begin{tabular}{lcc} 
& $I C U$ & 24 hours \\
\hline $\mathrm{PaO}_{2}(\mathrm{~mm} \mathrm{Hg}) / \mathrm{FiO}_{2}$ & $281.5 / 94$ & $252.2 / 89.7$ \\
$\mathrm{MPAP}(\mathrm{mm} \mathrm{Hg})$ & $25 \pm 7.3$ & $23 \pm 7$ \\
$\mathrm{CO}(\mathrm{L})$ & $4.5 \pm 0.8$ & $5.2 \pm 1.1$ \\
$\mathrm{PVR}\left(\right.$ dynes $\left.\cdot \mathrm{sec} \cdot \mathrm{cm}^{-5}\right)$ & $294.5 \pm 218.2$ & $183.8 \pm 108.8$ \\
$\mathrm{SVR}\left(\right.$ dynes $\left.\cdot \mathrm{sec} \cdot \mathrm{cm}^{-5}\right)$ & $1308 \pm 619.9$ & $1262 \pm 278.6$
\end{tabular}

$\mathrm{ICU}$, Intensive care unit; $\mathrm{PaO}_{2}$, pulmonary arterial oxygen tension; $\mathrm{FiO}_{2}$, inspired oxygen fraction; $M P A P$, mean PA pressure; $C O$, cardiac output; $P V R$, pulmonary vascular resistance; SVR, systemic vascular resistance.

and retrograde instillation of the preservation solution through the pulmonary veins occurs at the back table $(250$ $\mathrm{ml}$ in each vein; Fig. 2). The results have been satisfactory either way. The early lung allograft function is shown in Table I. Mechanical ventilation was $24.3 \pm 22.6$ hours for unilateral lung transplantation and $34.3 \pm 31.9$ hours for bilateral lung transplantation.

Comment. The pulmonary venous circulatory system is a low-resistance, high-capacity network. Flushing of preservation fluid through this system is straightforward and results in rapid and uniform distribution of the solution. Timing for administration of the pneumoplegic solution does not hamper delivery of the cardioplegic solution, and both can be flushed at the same time or once the heart has been arrested, because most of the solution exits through the PA. We followed the courses of 18 hearts retrieved by different surgical teams and the five retrieved by our own team, and no primary heart graft failures were documented. Furthermore, it was previously shown in three cases of heart-lung transplantation by Sarsam and cowork$\mathrm{ers}^{5}$ that no left ventricular dilatation occurs despite transatrial cannulation for preservation.
The bronchial circulation is also known as the pulmonary systemic network. It supplies not only the extrapulmonary and intrapulmonary airway system but also the neurovascular bundles, lymphoid structures, and visceral pleura. Bronchial veins arising from the intrapulmonary airway and parenchymal systems drain into the left side of the heart through the pulmonary veins. The real implications of bronchial-pulmonary preservation are still unclear, however, and remain to be elucidated.

In conclusion, this combination of retrograde and antegrade preservation, or the other way around, has resulted in satisfactory early graft function. It is currently a mainstay of our clinical lung transplantation program protocol.

\section{REFERENCES}

1. Baretti R, Bitu-Moreno J, Friedhelm B, Matheis G, Franchischetti Y, Kreitmayr B. Distribution of lung preservation solutions in parenchyma and airways: influence of atelectasis and route of delivery. J Heart Lung Transplant 1995;14: 80-91.

2. Chen CZ, Gallagher RC, Ardery P, Dyckman W, Low HBC. Retrograde versus antegrade flush in canine left lung preservation for six hours. J Heart Lung Transplant 1996;15:395-403.

3. Varela A, Montero CG, Córdoba M, Antequera A, Pérez M, Tabuenca MJ, et al. Improved distribution of pulmonary flush solution to the tracheobronchial wall in pulmonary transplantation. Eur Surg Res 1997;29:1-4.

4. Varela A, Montero CG, Córdoba M, Serrano-Fiz S, Burgos R, Téllez JC, et al. Clinical experience with retrograde lung preservation. Transplant Int 1996;9(Suppl 1):S296-8.

5. Sarsam MAI, Yonan NA, Deiraniya AK, Rahman AN. Retrograde pulmonaryplegia for lung preservation in clinical transplantation: a new technique. J Heart Lung Transplant 1993;12:494-8. 